\title{
A Comparative Study of Three Resolving Parameters of Graphs
}

\author{
Hafiz Muhammad Ikhlaq $\mathbb{D}^{1},{ }^{1}$ Hafiz Muhammad Afzal Siddiqui $(\mathbb{D}){ }^{1}$ \\ and Muhammad Imran $\mathbb{1}^{2}$ \\ ${ }^{1}$ Department of Mathematics, COMSATS University Islamabad (CUI), Lahore Campus, Lahore 54000, Pakistan \\ ${ }^{2}$ Department of Mathematical Sciences, United Arab Emirates University, P. O. Box, Al Ain 15551, UAE \\ Correspondence should be addressed to Muhammad Imran; imrandhab@gmail.com
}

Received 20 September 2021; Accepted 22 November 2021; Published 15 December 2021

Academic Editor: Jorge-Antonio Lopez-Renteria

Copyright (c) 2021 Hafiz Muhammad Ikhlaq et al. This is an open access article distributed under the Creative Commons Attribution License, which permits unrestricted use, distribution, and reproduction in any medium, provided the original work is properly cited.

\begin{abstract}
Graph theory is one of those subjects that is a vital part of the digital world. It is used to monitor the movement of robots on a network, to debug computer networks, to develop algorithms, and to analyze the structural properties of chemical structures, among other things. It is also useful in airplane scheduling and the study of diffusion mechanisms. The parameters computed in this article are very useful in pattern recognition and image processing. A number $d(f, w)=\min \{d(w, t), d(w, s)\}$ is referred as distance between $f=t s$ an edge and $w$ a vertex. $d\left(w, f_{1}\right) \neq d\left(w, f_{2}\right)$ implies that two edges $f_{1}, f_{2} \in E$ are resolved by node $w \in V$. A set of nodes $A$ is referred to as an edge metric generator if every two links/edges of $\Gamma$ are resolved by some nodes of $A$ and least cardinality of such sets is termed as edge metric dimension, edim $(\Gamma)$ for a graph $\Gamma$. A set $B$ of some nodes of $\Gamma$ is a mixed metric generator if any two members of $V \cup E$ are resolved by some members of $B$. Such a set $B$ with least cardinality is termed as mixed metric dimension, $m \operatorname{dim}(\Gamma)$. In this paper, the metric dimension, edge metric dimension, and mixed metric dimension of dragon graph $T_{n, m}$, line graph of dragon graph $L\left(T_{n, m}\right)$, paraline graph of dragon graph $L\left(S\left(T_{n, m}\right)\right)$, and line graph of line graph of dragon graph $L\left(L\left(T_{n, m}\right)\right)$ have been computed. It is shown that these parameters are constant, and a comparative analysis is also given for the said families of graphs.
\end{abstract}

\section{Introduction}

For several years, the characteristics associated to graph distances have piqued the interest of various scholars, and one of them, the metric dimension, has recently been the focus of them. The theory of metric dimension was given by Slater in 1975 [1] and this theory was further elaborated as resolving set of graphs by Harary and Melter in 1976 [2]. In 2003, Brigham et al. [3] determined resolving dominating set and resolving domination number in graphs. In 2003, Chartrand et al. [4] studied the independent resolving set in nontrivial connected graphs of order $n$. The order of minimal independent resolving set is known as independent resolving number and is denoted as $i r(\Gamma)$. In 2003, Saenpholphat and Zhang [5] calculated the connected resolving number of complete graph $K_{n}$, star graph $K_{1, n-1}$, and Cartesian products of $\Gamma \times K_{2}$. In 2007, Oellermann and Peters-Fransen [6] found the strong metric dimension of graphs and digraphs. In 2010, Okamoto et al. [7] discussed the local metric dimension of graphs and some bounds of it. In 2017, Yero et al. [8] determined the k-metric dimensional graphs. In 2016, Imran and Siddiqui [9] computed the metric dimension of some convex polytopes generated by wheel related graphs. A graph in which all vertices have the same degree is called a regular graph. Ali presented a survey of antiregular graphs [10], and he gathered the known results concerning the antiregular graphs.

There are many applications of this parameter to robot navigation in networks which have been discussed in [11] and applications to chemistry have been discussed in $[4,12]$. Furthermore, this issue has certain applications to pattern recognition and image processing difficulties, some of which require the usage of hierarchical data structures [13]. Some interesting connections between metric generators in graphs and the master mind game or coin weighing have been presented in [14]. The metric dimension of infinite graphs 
was studied in [15], and extremal graphs for metric dimension and diameter were considered in [16].

Let $\Gamma$ be a simple connected and undirected graph with the vertex set $V(\Gamma)$ and edge set $E(\Gamma)$. The distance between any two vertices $u, v \in V(\Gamma)$ is denoted as $d(u, v)$ and is defined as the length of the shortest path between $u$ and $v$. The vertex $t$ resolves the vertices $u$ and $v$ if $d(t, u) \neq d(t, v)$. An ordered set of $i$ vertices $R=\left\{x_{1}, x_{2}, \ldots, x_{i}\right\}$; the identification of $x$ depending on $R$ is the order $i-$ tuple and is written as

$$
r(u \mid R)=\left(d\left(x, x_{1}\right), d\left(x, x_{2}\right), d\left(x, x_{3}\right), \ldots, d\left(x, x_{i}\right)\right) .
$$

If the different vertices of $\Gamma$ have different codes based on $R$, then $R$ is known as a resolving set for $\Gamma$. Let $\Omega \subset \Gamma$ of graph $\Gamma$ be such that $|\Omega|=\min \{|R|: \mathrm{R}$ is a resolving set for $\Gamma\}$, then $\Omega$ is a metric basis for the $g r a p h ~ \Gamma$ and $\operatorname{dim}(\Gamma)=|\Omega|$ is metric dimension of the graph $\Gamma$.

Recently, the idea of edge metric dimension of graph $(e \operatorname{dim}(\Gamma))$ was given by Kelenc et al. [17], and they also presented some results and comparison of metric dimension and edge metric dimension for some well-known families of graphs like path graph $P_{n}, e \operatorname{dim}\left(P_{n}\right)=\operatorname{dim}\left(P_{n}\right)=1$ where $n$ is number of vertices of graph, cycle graph $C_{n}$ : $e \operatorname{dim}\left(C_{n}\right)=\operatorname{dim}\left(C_{n}\right)=2$, complete graph $K_{n}: \operatorname{edim}\left(K_{n}\right)$ $=\operatorname{dim}\left(K_{n}\right)=n-1$, and complete bipartite graph $K_{(r, t)}$ : $\operatorname{edim}\left(K_{(r, t)}\right)=\operatorname{dim}\left(K_{(r, t)}\right)=r+t-2$, where $r, t>1$ are vertices of each partite set; if $\Gamma$ is a grid graph $\Gamma=P_{r} \times P_{t}$ with $r \geq t \geq 2$, then $e \operatorname{dim}(\Gamma)=2$. Furthermore, they have raised many open problems linked to the nature of metric dimension and edge metric dimension. In 2018, Liu et al. [18] worked on edge version of metric dimension and calculated edge metric dimension of necklace graph and line graph of necklace graph. In 2021, Deng et al. [19] discussed the edge resolvability parameter for different families of Mobius ladder networks, and they find the exact edge metric dimension of triangular, square, and hexagonal Mobius ladder networks.

The distance between an edge $e=t p$ and a vertex $q$ is denoted as $d(e, q)$ and is defined as $d(e, q)=\min \{d(t, q), d(p, q)\}$. The vertex $u$ resolves the edges $e_{i}$ and $e_{l}$ if $d\left(e_{i}, u\right) \neq d\left(e_{l}, u\right)$. An ordered set of $j$ vertices $A=\left\{a_{1}, a_{2}, \ldots, a_{j}\right\}$; the identification of an edge $e_{\alpha}$ depending on $A$ is the order $j$-tuple and is written as

$$
r\left(e_{\alpha} \mid A\right)=\left(d\left(e_{\alpha}, a_{1}\right), d\left(e_{\alpha}, a_{2}\right), d\left(e_{\alpha}, a_{3}\right), \ldots, d\left(e_{\alpha}, a_{j}\right)\right) .
$$

If the different edges of $\Gamma$ have different codes based on $A$, then $A$ is known as an edge resolving set for $\Gamma$. Let $L$ be a subset of vertex set of graph $\Gamma$ such that $|L|=\min \{|L|: L$ is an edge resolving set for $\Gamma\}$, then $L$ is an edge metric basis for the graph $\Gamma$ and $\operatorname{edim}(\Gamma)=|L|$ is edge metric dimension of the graph $\Gamma$.

Kelenc et al. [20] has presented the idea of mixed metric dimension and denoted it as $m \operatorname{di} m(\Gamma)$, and they presented the mixed metric dimension of path graph $P_{n}$, $m \operatorname{dim}\left(P_{n}\right)=2$, cycle graph $C_{n}, m \operatorname{dim}\left(C_{n}\right)=3$, and complete bipartite graph $K_{(r, t)}, m \operatorname{dim}\left(K_{(r, t)}\right)=r+t-1$ where $r, t=2$; otherwise, $m \operatorname{dim}\left(K_{(r, t)}\right)=r+t-2$ and grid graph
$\Gamma=P_{r} \square P_{t}, m \operatorname{dim}(\Gamma)=3$ where $r \geq t \geq 2$. An ordered subset of a vertex set of a graph is called mixed resolving set if it is both vertex resolving set and edge resolving set. Let $\Gamma$ be a graph and $X=x_{1}, x_{2}, \ldots, x_{k}$ be an ordered subset of vertices of graph $\Gamma$. If all vertices and edges of $\Gamma$ have different codes of representation with respect to the set $X$, then $X$ is known as a mixed resolving set for graph $\Gamma$. Let $M$ be a subset of vertex set of graph $\Gamma$ such that $|M|=\min$ $\{: X$ is a mixed resolving set for graph $\Gamma\}$. Then, $M$ is known as a mixed metric basis for $\Gamma$, and $m \operatorname{dim}(\Gamma)=|M|$ is a mixed metric dimension of the graph $\Gamma$. In 2016, Yero [21] presented some bounds or closed formulae for the (edge, mixed) metric dimension of several families of graphs.

Figure 1 represents graph $\Gamma$ with $V(\Gamma)=\left\{v_{1}, v_{2}, \ldots, v_{8}\right\}$, and $E(\Gamma)=\{a, b, c, d, e, f, g, h\}$ are vertex set and edge set, respectively. The set $A=\left\{v_{2}, v_{7}\right\}$ is an edge resolving set of $\Gamma$. The representation of all edges with respect to $A$ is as given in Table 1.

All edges have different representations with respect to $A$, so $e \operatorname{dim}(\Gamma)=2$.

The set $B=\left\{v_{2}, v_{7}, v_{8}\right\}$ is a mixed resolving set of $\Gamma$. The representation of all edges and vertices with respect to $B$ is as given in Table 2 .

All edges and vertices have different representations with respect to $B$, so $m \operatorname{dim}(\Gamma)=3$.

Theorem 1 (see [12]). A connected graph $\Gamma$ of order $n$ has dimension 1 if and only if $\Gamma=P_{n}$.

Theorem 2 (see [22]). Let $T_{n, m}$ be a dragon graph for $n \geq 3$ and $m \geq 2$. Then, $\operatorname{dim}\left(T_{n, m}\right)=2$.

Theorem 3 (see [17]). The edge metric dimension of a graph $G$ is 1 if and only if $\Gamma$ is a path.

Theorem 4 (see [20]). The mixed metric dimension of a graph $G$ is 2 if and only if $\Gamma$ is a path.

\section{Results on Dragon Graph $T_{n, m}$}

Dragon graph is obtained by identifying vertex $v_{n}$ of cycle graph $C_{n}$ with vertex $u_{0}$ of path graph $P_{m+1}$ and is denoted as $T_{n, m}$. Order of dragon graph $T_{n, m}$ is $n+m$. Its vertex set is $V\left(T_{n, m}\right)=\left\{v_{1}, v_{2}, \ldots, v_{n}, u_{1}, u_{2}, \ldots, u_{m}\right\}$ and edge set is $E\left(T_{n, m}\right)=\left\{v_{i} v_{i+1}: 1 \leq i \leq n\right\} \cup\left\{u_{j} u_{j+1} 1 \leq j \leq m-1\right\} \cup\left\{v_{n} u_{1}\right\}$ where $v_{n+1}=v_{1}$ as shown in Figure 2 .

Theorem 5. Let $T_{n, m}$ be a dragon graph for $n \geq 3$ and $m \geq 2$. Then, $\operatorname{edim}\left(T_{n, m}\right)=2$.

Proof. Since dragon graph is not a path graph, $e \operatorname{dim}\left(T_{n, m}\right) \geq 2$. In this case, $A=\left\{v_{1}, u_{m}\right\}$ is an edge resolving set of $T_{n, m}$. All edges are labeled as

$$
\begin{aligned}
e_{i} & =\left\{v_{i} v_{i+1}: 1 \leq i \leq n\right\}, \\
e_{j+1}^{\prime} & =\left\{u_{j} u_{j+1}: 1 \leq j \leq m-1\right\}, \\
e_{1}^{\prime} & =v_{n} u_{1} .
\end{aligned}
$$




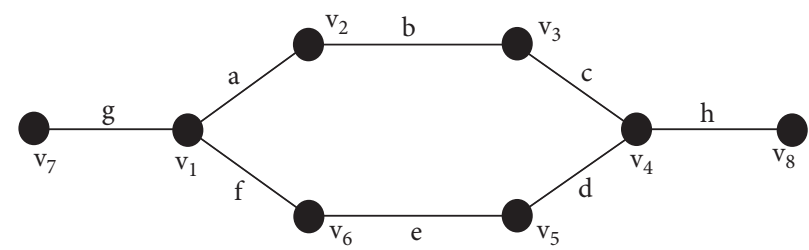

Figure 1: A graph with the $\operatorname{edim}(\Gamma)=2$.

TABle 1: Edge distance codes of $\Gamma$ w.r.t. $A$.

\begin{tabular}{lcccc}
\hline$d(.,)$. & $a$ & $b$ & $c$ & $d$ \\
\hline$A=\left\{v_{2}, v_{7}\right\}$ & $(0,1)$ & $(0,2)$ & $(1,3)$ & $(2,3)$ \\
$d(.,)$. & $e$ & $f$ & $g$ & $h$ \\
$A=\left\{v_{2}, v_{7}\right\}$ & $(2,2)$ & $(1,1)$ & $(1,0)$ & $(2,4)$ \\
\hline
\end{tabular}

TABLE 2: Mixed distance codes of $\Gamma$ w.r.t. $B$.

\begin{tabular}{lcccc}
\hline$d(.,)$. & $a$ & $b$ & $c$ & $d$ \\
\hline$B=\left\{v_{2}, v_{7}, v_{8}\right\}$ & $(0,1,3)$ & $(0,2,2)$ & $(1,3,1)$ & $(2,3,1)$ \\
$d(.,)$. & $e$ & $f$ & $g$ & $h$ \\
$B=\left\{v_{2}, v_{7}, v_{8}\right\}$ & $(2,2,2)$ & $(1,1,3)$ & $(1,0,4)$ & $(2,4,0)$ \\
$d(.,)$. & $v_{1}$ & $v_{2}$ & $v_{3}$ & $v_{4}$ \\
$B=\left\{v_{2}, v_{7}, v_{8}\right\}$ & $(1,1,4)$ & $(0,2,3)$ & $(1,3,2)$ & $(2,4,1)$ \\
$d(.,)$. & $v_{5}$ & $v_{6}$ & $v_{7}$ & $v_{8}$ \\
$B=\left\{v_{2}, v_{7}, v_{8}\right\}$ & $(3,3,2)$ & $(2,2,3)$ & $(1,1,4)$ & $(2,0,5)$ \\
\hline
\end{tabular}

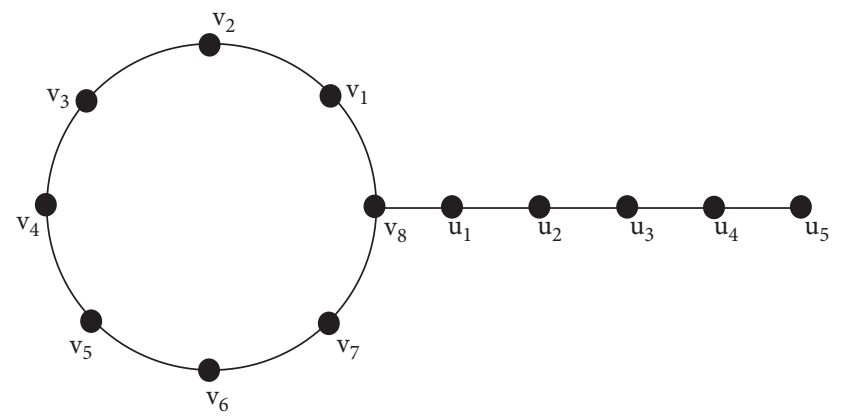

Figure 2: Dragon graph $T_{8,5}$.

The representations of all edges with respect to $A$ are as follows:

$$
r\left(e_{i} \mid A\right)= \begin{cases}(i-1, m+i) & 1 \leq i \leq\left\lfloor\frac{n}{2}\right\rfloor-1 ; \\ (i-1, m+n-i-1) & i=\left\lfloor\frac{n}{2}\right\rfloor \\ (n-i, m+n-i-1) & \left\lfloor\frac{n}{2}\right\rfloor+1 \leq i \leq n-1 ; \\ (0, m) & i=n .\end{cases}
$$$$
r\left(e_{j}^{\prime} \mid A\right)=(j, m-j): 1 \leq j \leq m .
$$

All edges have distinguished representation, and this fact can easily be verified, which implies that $e \operatorname{dim}\left(T_{n, m}\right) \leq 2$. So, we obtained $e \operatorname{dim}\left(T_{n, m}\right)=2$.

Theorem 6. Let $T_{n, m}$ be a dragon graph for $n \geq 3$ and $m \geq 2$. Then, $m \operatorname{dim}\left(T_{n, m}\right)=3$.

Proof. Since dragon graph is not a path graph, $m \operatorname{dim}\left(T_{n, m}\right) \geq 3$. All edges are labeled as

$$
\begin{aligned}
e_{i} & =\left\{v_{i} v_{i+1}: 1 \leq i \leq n\right\}, \\
e_{j+1}^{\prime} & =\left\{u_{j} u_{j+1}: 1 \leq j \leq m-1\right\}, \\
e_{1}^{\prime} & =v_{n} u_{1} .
\end{aligned}
$$

In this case, $A=\left\{v_{1}, v_{[n / 2]}, u_{m}\right\}$ is mixed resolving set of $T_{n, m}$. The representations of all vertices with respect to $A$ are as follows:

$$
r\left(u_{j} \mid A\right)=\left(j+1,\left\lfloor\frac{n}{2}\right\rfloor+j, m-j\right): 1 \leq j \leq m .
$$

For $n$ even,

$$
r\left(v_{i} \mid A\right)= \begin{cases}\left(i-1, \frac{n}{2}-i, m+i\right) & 1 \leq i \leq \frac{n}{2} \\ \left(n-i+1, i-\frac{n}{2}, m+n-i\right) & \frac{n}{2}+1 \leq i \leq n .\end{cases}
$$

For $n$ odd,

$$
r\left(v_{i} \mid A\right)= \begin{cases}\left(i-1,\left\lceil\frac{n}{2}\right\rceil-i, m+i\right) & 1 \leq i \leq\left\lfloor\frac{n}{2}\right\rfloor ; \\ \left(i-1,\left\lceil\frac{n}{2}\right\rceil-i, m+n-i\right) & i=\left\lfloor\frac{n}{2}\right\rfloor+1 ; \\ \left(n-i+1, i-\left\lceil\frac{n}{2}\right\rceil, m+n-i\right) & \left\lfloor\frac{n}{2}\right\rfloor+2 \leq i \leq n .\end{cases}
$$

The representations of all edges with respect to $A$ are as follows:

$$
r\left(e_{j}^{\prime} \mid A\right)=\left(j,\left\lfloor\frac{n}{2}\right\rfloor+j-1, m-j\right): 1 \leq j \leq m .
$$

For $n$ even, 
$r\left(e_{i} \mid A\right)= \begin{cases}\left(i-1, \frac{n}{2}-i-1, m+i\right) & 1 \leq i \leq \frac{n}{2}-1 ; \\ \left(i-1, i-\frac{n}{2}, m+n-i-1\right) & i=\frac{n}{2} ; \\ \left(n-i, i-\frac{n}{2}, m+n-i-1\right) & \frac{n}{2}+1 \leq i \leq n-1 ; \\ \left(0, \frac{n}{2}-1, m\right) & i=n .\end{cases}$

For $n$ odd,

$$
r\left(e_{i} \mid A\right)= \begin{cases}\left(i-1,\left\lceil\frac{n}{2}\right\rceil-i-1, m+i\right) & 1 \leq i \leq\left\lfloor\frac{n}{2}\right\rfloor-1 ; \\ \left(i-1,\left\lceil\frac{n}{2}\right\rceil-i-1, m+n-i-1\right) & i=\left\lfloor\frac{n}{2}\right\rfloor ; \\ \left(n-i, i-\left\lceil\frac{n}{2}\right\rceil, m+n-i-1\right) & \left\lfloor\frac{n}{2}\right\rfloor+1 \leq i \leq n-1 ; \\ \left(0,\left\lceil\frac{n}{2}\right\rceil-1, m\right) & i=n .\end{cases}
$$

From the above representation, it is clear that no two vertices, edges, and an edge or a vertex of $T_{n, m}$ have the same representation which implies that $m \operatorname{dim}\left(T_{n, m}\right) \leq 3$. So, we obtained $m \operatorname{dim}\left(T_{n, m}\right)=3$.

\section{Results on Line Graph of Dragon \\ Graph $L\left(T_{n, m}\right)$}

A line graph $L(\Gamma)$ of $\Gamma$ can be generated by assuming edge set of $\Gamma$ as vertex set of $L(\Gamma)$, and any two vertices of $L(\Gamma)$ are adjacent if and only if they are neighboring edges in $\Gamma$. Line graph of dragon graph has $V\left(L\left(T_{n, m}\right)\right)=\left\{a_{1}, a_{2}, \ldots, a_{n}\right.$, $\left.b_{1}, b_{2}, \ldots, b_{m}\right\}$ and $E\left(L\left(T_{n, m}\right)\right)=\left\{a_{i}, a_{i+1}, b_{j} b_{j+1}: 1 \leq i \leq n\right.$, $1 \leq j \leq m-1\} \cup\left\{a_{1} b_{1}, a_{n} b_{1}\right\}$ as its set of vertices and set of edges, where $a_{n+1}=a_{1}$ as shown in Figure 3 .

Theorem 7. Let $L\left(T_{n, m}\right)$ be a line graph of dragon graph for $n \geq 3$ and $m \geq 2$. Then, $\operatorname{dim}\left(L\left(T_{n, m}\right)\right)=2$.

Proof. Since $\operatorname{dim}\left(L\left(T_{n, m}\right)\right) \geq 2$, it is not a path graph $P_{n}$. $A=\left\{a_{2}, b_{m}\right\}$ is resolving set of $L\left(T_{n, m}\right)$, and the representations of all vertices with respect to $A$ are as follows:

$$
r\left(a_{i} \mid A\right)= \begin{cases}(1, m), & i=1 ; \\ (i-2, m+i-1) & 2 \leq i \leq\left\lceil\frac{n}{2}\right\rceil ; \\ (i-2, n+m-i) & i=\left\lceil\frac{n}{2}\right\rceil+1 ; \\ (n-i+2, n+m-i) & \left\lceil\frac{n}{2}\right\rceil+2 \leq i \leq n .\end{cases}
$$

$$
r\left(b_{j} \mid A\right)=(j+1, m-j): 1 \leq j \leq m .
$$

All vertices have different representation, which implies that $\operatorname{dim}\left(L\left(T_{n, m}\right)\right) \leq 2$. So, we obtained $\operatorname{dim}\left(L\left(T_{n, m}\right)\right)=2$.

Theorem 8. Let $L\left(T_{n, m}\right)$ be a line graph of dragon graph for $n \geq 3$ and $m \geq 2$. Then, $e$ di $m\left(L\left(T_{n, m}\right)\right)=2$.

Proof. Since edim $\left(L\left(T_{n, m}\right)\right) \geq 2$, it is not a path graph $P_{n}$. All edges of $L\left(T_{n, m}\right)$ are labeled as $e_{1}^{\prime}=a_{1} b_{1}$ and $e_{0}^{\prime}=a_{n} b_{1}$.

$e_{i}=\left\{a_{i} a_{i+1}: 1 \leq i \leq n\right\}$ and $e_{j+1}^{\prime}=\left\{b_{j} b_{j+1}: 1 \leq j \leq m-1\right\}$.

$A=\left\{a_{2}, b_{m}\right\}$ is an edge resolving set of $L\left(T_{n, m}\right)$, and representations of all edges with respect to $A$ are as follows:

$$
\begin{aligned}
& r\left(e_{0}^{\prime} \mid A\right)=(2, m-1), \\
& r\left(e_{j}^{\prime} \mid A\right)=(j, m-j): 1 \leq j \leq m, \\
& r\left(e_{i} \mid A\right)= \begin{cases}(0, m+i-1) & i=1 ; \\
(i-2, m+i-1) & i=\left\lfloor\frac{n}{2}\right\rfloor ; \\
(i-2, m+n-i-1) & \\
(n-i+1, m+n-i-1) & \left\lfloor\frac{n}{2}\right\rfloor+2 \leq i \leq n-1 ; \\
(n-i+1, m) & i=n .\end{cases}
\end{aligned}
$$

All edges have different representation, which implies that $\quad \operatorname{dim}\left(L\left(T_{n, m}\right)\right) \leq 2$. So, we obtained $\operatorname{edim}\left(L\left(T_{n, m}\right)\right)=2$.

Theorem 9. Let $L\left(T_{n, m}\right)$ be a line graph of dragon graph for $n \geq 3$ and $m \geq 1$. Then, $m \operatorname{dim}\left(L\left(T_{n, m}\right)\right)=3$. 


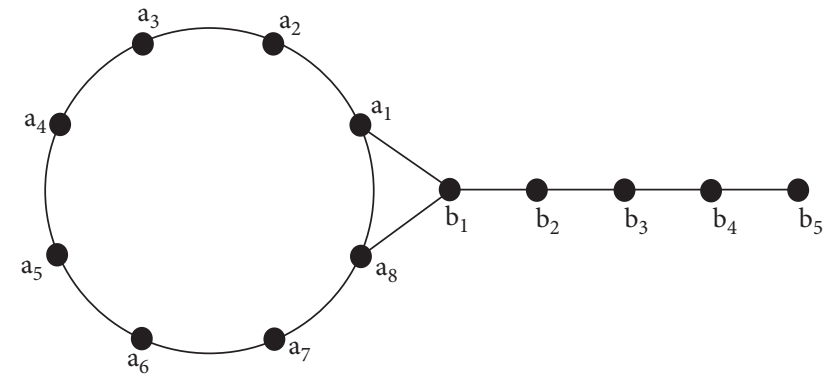

Figure 3: Line graph of dragon graph $L\left(T_{8,5}\right)$.

Proof. Since $m \operatorname{dim}\left(L\left(T_{n, m}\right)\right) \geq 3$, it is not a path graph $P_{n}$. All edges of $L\left(T_{n, m}\right)$ are labeled as

$$
\begin{aligned}
& e_{n}=a_{1} a_{n}, \\
& e_{1}^{\prime}=a_{1} b_{1}, \\
& e_{0}^{\prime}=a_{n} b_{1} .
\end{aligned}
$$

$e_{i}=\left\{a_{i} a_{i+1}: 1 \leq i \leq n-1\right\} \quad$ and $\quad e_{j+1}{ }^{\prime}=\left\{b_{j} b_{j+1}: 1 \leq j \leq\right.$ $m-1\}$.

$A=\left\{a_{2}, a_{\lfloor n / 2\rfloor+1}, b_{m}\right\}$ is mixed resolving set of $L\left(T_{n, m}\right)$, and the representations of all edges with respect to $A$ are as follows:

$$
\left.r\left(e_{i}\right\rfloor A\right)= \begin{cases}\left(0,\left\lfloor\frac{n}{2}\right\rfloor-i, m+i-1\right) & i=1 ; \\ \left(i-2,\left\lfloor\frac{n}{2}\right\rfloor-i, m+i-1\right) & 2 \leq i \leq\left\lfloor\frac{n}{2}\right\rfloor ; \\ \left(i-2, i-\left\lfloor\frac{n}{2}\right\rfloor-1, m+n-i-1\right) & i=\left\lfloor\frac{n}{2}\right\rfloor+1 ; \\ \left(n-i+1, i-\left\lfloor\frac{n}{2}\right\rfloor-1, m+n-i-1\right) & \left\lfloor\frac{n}{2}\right\rfloor+2 \leq i \leq n-1 ; \\ & i=n .\end{cases}
$$

For $n$ even,

$$
\begin{aligned}
& r\left(e_{j}^{\prime} \mid A\right)=\left\{\begin{array}{l}
\left(j, \frac{n}{2}, m-j\right): j=1 ; \\
\left(j, \frac{n}{2}+j-2, m-j\right): 2 \leq j \leq m,
\end{array}\right. \\
& r\left(e_{0}^{\prime} \mid A\right)=\left(2, \frac{n}{2}-1, m-1\right) .
\end{aligned}
$$

For $n$ odd,

$$
\begin{aligned}
& r\left(e_{j}^{\prime} \mid A\right)=\left(j,\left\lfloor\frac{n}{2}\right\rfloor+j-1, m-j\right): 1 \leq j \leq m, \\
& r\left(e_{0}^{\prime} \mid A\right)=\left(2,\left\lfloor\frac{n}{2}\right\rfloor, m-1\right) .
\end{aligned}
$$
follows:

$$
r\left(a_{p} \mid A\right)= \begin{cases}\left(1,\left\lfloor\frac{n}{2}\right\rfloor+1-p, m+p-1\right) & p=1 ; \\ \left(p-2,\left\lfloor\frac{n}{2}\right\rfloor+1-p, m+p-1\right) & 2 \leq p \leq\left\lceil\frac{n}{2}\right\rceil ; \\ \left(p-2, p-\left\lfloor\frac{n}{2}\right\rfloor-1, m+n-p\right) & p=\left\lceil\frac{n}{2}\right\rfloor+1 ; \\ \left(n-p+2, p-\left\lfloor\frac{n}{2}\right\rfloor-1, m+n-p\right) & \left\lceil\frac{n}{2}\right\rfloor+2 \leq p \leq n .\end{cases}
$$

$r\left(b_{q} \mid A\right)=\left(q+1,\left\lceil\frac{n}{2}\right\rceil+q-1, m-q\right): 1 \leq q \leq m$.

From the above representation, it is clear that no two vertices, edges, and an edge or a vertex of $L\left(T_{n, m}\right)$ have the same representation which implies that $m \operatorname{dim}\left(L\left(T_{n, m}\right)\right) \leq 3$. So, we obtained $m \operatorname{dim}\left(L\left(T_{n, m}\right)\right)=3$.

\section{Results on Paraline Graph of Dragon \\ Graph $\left.L\left(S\left(T_{n, m}\right)\right)\right)$}

Paraline graph is the line graph of the subdivision graph of any graph. In subdivision graph $S=S(\Gamma)$, the vertex set is $V(S)=V(\Gamma) \cup E(\Gamma)$ and the vertex of $S$ corresponding to the edge $u v$ of $\Gamma$ is inserted in the edge $u v$ of $\Gamma$. In line graph $L=L(\Gamma)$, the vertex set is $V(L)=E(G)$ and two vertices of $L$ are adjacent if the corresponding edges of $\Gamma$ are incident. Paraline graph of dragon graph has $V\left(L\left(S\left(T_{n, m}\right)\right)\right)=$ $\left\{a_{1}, a_{2}, \ldots, a_{2 n}, b_{1}, b_{2}, \ldots, b_{2 m}\right\} \quad$ and $E\left(L\left(S\left(T_{n, m}\right)\right)\right)=$ $\left\{a_{i}, a_{i+1}, b_{j} b_{j+1}: 1 \leq i \leq 2 n, 1 \leq j \leq 2 m-1\right\} \cup\left\{a_{1} b_{1}, a_{2 n} b_{1}\right\}$ as its set of vertices and set of edges, where $a_{2 n+1}=a_{1}$ as shown in Figure 4.

Theorem 10. Let $L\left(S\left(T_{n, m}\right)\right)$ be a paraline graph of dragon graph for $n \geq 3$ and $m \geq 2$. Then, $\operatorname{dim}\left(L\left(S\left(T_{n, m}\right)\right)\right)=2$.

Proof. Since $\operatorname{dim}\left(L\left(S\left(T_{n, m}\right)\right)\right) \geq 2$, it is not a path graph $P_{n}$. $A=\left\{a_{2}, b_{2 m}\right\}$ is resolving set of $L\left(S\left(T_{n, m}\right)\right)$, and the representations of all vertices with respect to $A$ are

$$
\begin{aligned}
& r\left(a_{i} \mid A\right)= \begin{cases}(1,2 m), & i=1 ; \\
(i-2,2 m+i-1) & 2 \leq i \leq n ; \\
(i-2,2(n+m)-i) & i=n+1 ; \\
(2 n-i+2,2(n+m)-i) & n+2 \leq i \leq 2 n,\end{cases} \\
& r\left(b_{j} \mid A\right)=(j+1,2 m-j): 1 \leq j \leq 2 m .
\end{aligned}
$$

All vertices have different representation, and this fact can easily be verified, which implies that $\operatorname{dim}\left(L\left(S\left(T_{n, m}\right)\right)\right)$ $\leq 2$. So, we obtained $\operatorname{dim}\left(L\left(S\left(T_{n, m}\right)\right)\right)=2$.

Theorem 11. Let $L\left(S\left(T_{n, m}\right)\right)$ be a paraline graph of dragon graph for $n \geq 3$ and $m \geq 2$. Then, edim $\left(L\left(S\left(T_{n, m}\right)\right)\right)=2$. 


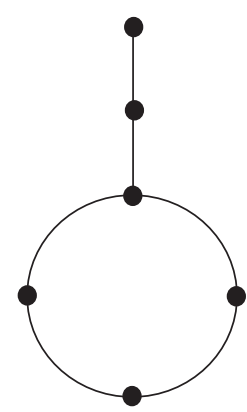

(a)

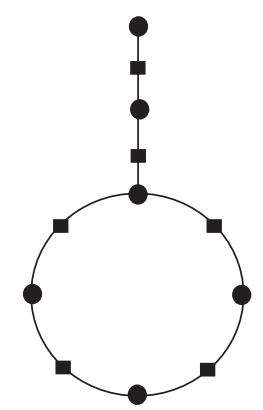

(b)

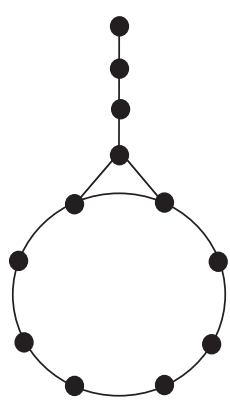

(c)

FIgURE 4: (a) Dragon graph $\left(T_{4,2}\right)$; (b) Subdivision graph of dragon graph $S\left(T_{4,2}\right)$; (c) Paraline graph of dragon graph $L\left(S\left(T_{4,2}\right)\right)$.

Proof. Since $e \operatorname{dim}\left(L\left(S\left(T_{n, m}\right)\right)\right) \geq 2$, it is not a path graph $P_{n}$. All edges of $L\left(S\left(T_{n, m}\right)\right)$ are labeled as $e_{1}^{\prime}=a_{1} b_{1}$ and $e_{0}^{\prime}=a_{2} n b_{1}$.

$e_{i}=\left\{a_{i} a_{i+1}: 1 \leq i \leq 2 n\right\} \quad$ and $\quad e_{j+1}^{\prime}=\left\{b_{j} b_{j+1}: 1 \leq j \leq\right.$ $2 m-1\}$.

$A=\left\{a_{2}, b_{2 m}\right\}$ is an edge resolving set of $L\left(S\left(T_{n, m}\right)\right)$, and representations of all edges with respect to $A$ are as follows:

$$
\begin{aligned}
& r\left(e_{0}^{\prime} \mid A\right)=(2,2 m-1), \\
& r\left(e_{j}^{\prime} \mid A\right)=(j, 2 m-j): 1 \leq j \leq m, \\
& r\left(e_{i} \mid A\right)= \begin{cases}(0,2 m+i-1) & i=1 ; \\
(i-2,2 m+i-1) & 2 \leq i \leq n ; \\
(i-2,2(m+n)-i-1) & i=n+1 ; \\
(2 n-i+1,2(m+n)-i-1) & n+2 \leq i \leq 2 n-1 ; \\
(2 n-i+1,2 m) & i=2 n .\end{cases}
\end{aligned}
$$

All edges have different representation, which implies that $e \operatorname{dim}\left(L\left(S\left(T_{n, m}\right)\right)\right) \leq 2$. So, we obtained $e \operatorname{dim}(L(S$ $\left.\left.\left(T_{n, m}\right)\right)\right)=2$.

Theorem 12. Let $L\left(S\left(T_{n, m}\right)\right)$ be a paraline graph of dragon graph for $n \geq 3$ and $m \geq 1$. Then, $m \operatorname{dim}\left(L\left(S\left(T_{n, m}\right)\right)\right)=3$.

Proof. Since $m \operatorname{dim} m\left(L\left(S\left(T_{n, m}\right)\right)\right) \geq 3$, it is not a path graph $P_{n}$. All edges of $L\left(S\left(T_{n, m}\right)\right)$ are labeled as

$$
\begin{aligned}
e_{2 n} & =a_{1} a_{2 n}, \\
e_{1}^{\prime} & =a_{1} b_{1}, \\
e_{0}^{\prime} & =a_{2 n} b_{1} .
\end{aligned}
$$

$e_{i}=\left\{a_{i} a_{i+1}: 1 \leq i \leq 2 n-1\right\}$ and $e_{j+1}^{\prime}=\left\{b_{j} b_{j+1}: 1 \leq j \leq\right.$ $2 m-1\}$.

$A=\left\{a_{2}, a_{n+1}, b_{2 m}\right\}$ is mixed resolving set of $L\left(S\left(T_{n, m}\right)\right)$, and the representations of all edges with respect to $A$ are as follows:

$$
r\left(e_{i} \mid A\right)= \begin{cases}(0, n-i, 2 m+i-1) & i=1 ; \\ (i-2, n-i, 2 m+i-1) & 2 \leq i \leq n ; \\ (i-2, i-n-1,2(m+n)-i-1) & i=n+1 ; \\ (2 n-i+1, i-n-1,2(m+n)-i-1) & n+2 \leq i \leq 2 n-1 ; \\ (2 n-i+1, i-n-1,2 m) & i=2 n .\end{cases}
$$$$
r\left(e_{j}^{\prime} \mid A\right)= \begin{cases}\left(2, \frac{n}{2}-1, m-1\right) & j=0 ; \\ (j, n, 2 m-j) & j=1 ; \\ (j, n+j-2,2 m-j) & 2 \leq j \leq 2 m .\end{cases}
$$

The representations of all vertices with respect to $A$ are as follows:

$$
\begin{aligned}
& r\left(a_{p} \mid A\right)= \begin{cases}(1, n+1-p, 2 m+p-1) & p=1 ; \\
(p-2, n+1-p, 2 m+p-1) & 2 \leq p \leq n ; \\
(p-2, p-n-1,2(m+n)-p) & p=n+1 ; \\
(2 n-p+2, p-n-1,2(m+n)-p) & n+2 \leq p \leq 2 n,\end{cases} \\
& r\left(b_{q} \mid A\right)=(q+1, n+q-1,2 m-q): 1 \leq q \leq 2 m .
\end{aligned}
$$

From the above representation, it is clear that no two vertices, edges, and an edge or a vertex of $L\left(S\left(T_{n, m}\right)\right)$ have the same representation which implies that $m \operatorname{dim}(L(S$ $\left.\left.\left(T_{n, m}\right)\right)\right) \leq 3$. So, we obtained $m \operatorname{dim}\left(L\left(S\left(T_{n, m}\right)\right)\right)=3$.

\section{Results on Line Graph of Dragon \\ Graph $L\left(L\left(T_{n, m}\right)\right)$}

Let $\Gamma$ be a graph and $L\left(L\left(T_{n, m}\right)\right)$ be line graph of line graph of dragon graph $T_{n . m}$. Vertex set of line graph of line graph of dragon graph is $V L\left(\left(L\left(T_{n, m}\right)\right)\right)=\left\{a_{1}, a_{2}, \ldots, a_{n}, b_{0}\right.$, $\left.b_{1}, b_{2}, \ldots, b_{m-1}\right\}$, and its edge set is $E\left(L\left(T_{n, m}\right)\right)=$ $\left\{a_{i}, a_{i+1}, b_{j} b_{j+1}: 1 \leq i \leq n, 0 \leq j \leq m-2\right\} \cup\left\{a_{n-1} b_{0}, a_{n} b_{0}, a_{n} b_{1}\right.$, $\left.a_{1} b_{1}, b_{0} b_{2}\right\}$ where $a_{n+1}=a_{1}$ as shown in Figure 5 . 


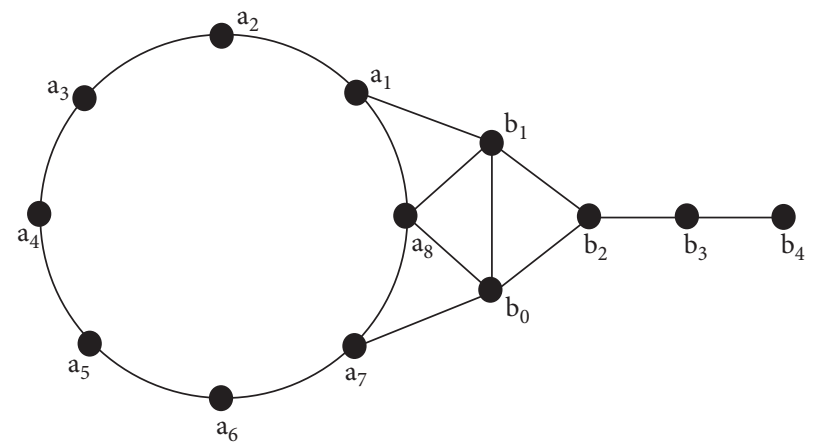

FIGURE 5: Paraline graph of dragon graph $L\left(L\left(T_{8,5}\right)\right)$.

Theorem 13. Let $L\left(L\left(T_{n, m}\right)\right)$ be a line graph of line graph of dragon graph for $n \geq 3$ and $m \geq 3$. Then, $\operatorname{dim} L\left(\left(L\left(T_{n, m}\right)\right)\right)=2$.

Proof. Since $\operatorname{dim} L\left(\left(L\left(T_{n, m}\right)\right)\right) \geq 2$, it is not a path graph $P_{n}$. $A=\left\{a_{2}, b_{m-1}\right\}$ is resolving set of $L\left(L\left(T_{n, m}\right)\right)$, and the representations of all vertices with respect to $A$ are as follows:

$$
r\left(b_{j} \mid A\right)= \begin{cases}(3, m-2) & j=0 \\ (j+1, m-j-1) & 1 \leq j \leq m-1 .\end{cases}
$$

For $n$ even,

$$
r\left(a_{i} \mid A\right)= \begin{cases}(1, m-1) & i=1 ; \\ (i-2, m+i-2) & i \leq \frac{n}{2} ; \\ (i-2, n+m-i-2) & \frac{n}{2}+1 ; \\ (n-i+2, n+m-i-2) & \frac{n}{2}+2 \leq n-1 ; \\ (2, m-1) & i=n .\end{cases}
$$

For $n$ odd,

$$
r\left(a_{i} \mid A\right)= \begin{cases}(1, m-1) & i=1 ; \\ (i-2, m+i-2) & 2 \leq i \leq\left\lfloor\frac{n}{2}\right\rfloor ; \\ (i-2, n+m-i-2) & \left\lfloor\frac{n}{2}\right\rfloor+1 \leq i \leq\left\lfloor\frac{n}{2}\right\rfloor+2 ; \\ (n-i+2, n+m-i-2) & \left\lfloor\frac{n}{2}\right\rfloor+3 \leq i \leq n-1 ; \\ (2, m-1) & i=n .\end{cases}
$$

All vertices have different representations, which imply that $\operatorname{dim}\left(L\left(L\left(T_{n, m}\right)\right)\right) \leq 2$. So, we obtained $\operatorname{dim}(L(L$ $\left.\left.\left(T_{n, m}\right)\right)\right)=2$.

Theorem 14. Let $L\left(L\left(T_{n, m}\right)\right)$ be line graph of line graph of dragon graph for $n \geq 5$ and $m \geq 3$. Then, edim $L$ $\left(\left(L\left(T_{n, m}\right)\right)\right)=4$.

Proof. Since $\operatorname{edim} L\left(\left(L\left(T_{n, m}\right)\right)\right) \geq 2$, it is not a path graph $P_{n}$. All edges are labeled as $e_{i}=\left\{a_{i} a_{i+1}: 1 \leq i \leq n\right\}$ and $e_{j}^{\prime}=\left\{b_{j} b_{j+1}: 0 \leq j \leq m-2\right\}$.

$$
\begin{aligned}
& e_{1}^{\prime \prime}=a_{n-1} b_{0}, \\
& e_{2}^{\prime \prime}=a_{n} b_{0}, \\
& e_{3}^{\prime \prime}=a_{n} b_{1}, \\
& e_{4}^{\prime \prime}=a_{1} b_{1}, \\
& e_{5}^{\prime \prime}=b_{0} b_{2} .
\end{aligned}
$$

$A=\left\{a_{2}, b_{0}, b_{1}, b_{m-1}\right\}$ is an edge resolving set of $L\left(L\left(T_{n, m}\right)\right)$, and the representations of all edges with respect to $A$ are as follows:

$$
\begin{aligned}
& r\left(e_{j}^{\prime} \mid A\right)= \begin{cases}(2,0,0, m-2), & j=0 ; \\
(2,1,0, m-3), & j=1 ; \\
(j+1, j-1, j-1, m-j-2), & 2 \leq j \leq m-2,\end{cases} \\
& r\left(e_{1}^{\prime \prime} \mid A\right)=(3,0,1, m-2), \\
& r\left(e_{2}^{\prime \prime} \mid A\right)=(2,0,1, m-2), \\
& r\left(e_{3}^{\prime \prime} \mid A\right)=(2,1,0, m-2), \\
& r\left(e_{4}^{\prime \prime} \mid A\right)=(1,1,0, m-2), \\
& r\left(e_{5}^{\prime \prime} \mid A\right)=(3,0,1, m-3) .
\end{aligned}
$$

For $n$ even, 


$$
r\left(e_{i} \mid A\right)= \begin{cases}(0,2,1, m-1) & i=1 ; \\ (i-2, i+1, i, m+i-2) & 2 \leq i \leq \frac{n}{2}-1 ; \\ (i-2, n-i-1, n-i, n+m-i-3) & \frac{n}{2} \leq i \leq \frac{n}{2}+1 ; \\ (n-i+1, n-i-1, n-i, n+m-i-3) & \frac{n}{2}+2 \leq i \leq n-2 ; \\ (2,1,1, m-1) & i=n-1 ; \\ (1,1,1, m-1) & i=n .\end{cases}
$$

For $n$ odd,

$$
r\left(e_{i} \mid A\right)= \begin{cases}(0,2,1, m-1) & i=1 ; \\ (i-2, i+1, i, m+i-2) & 2 \leq i \leq\left\lfloor\frac{n}{2}\right\rfloor-1 ; \\ (i-2, n-i-1, n-i-1, n+m-i-3) & i=\left\lfloor\frac{n}{2}\right\rfloor ; \\ (i-2, n-i-1, n-i, n+m-i-3) & i=\left\lfloor\frac{n}{2}\right\rfloor+1 ; \\ (n-i+1, n-i-1, n-i, n+m-i-3) & \left\lfloor\frac{n}{2}\right\rfloor+2 \leq i \leq n-2 ; \\ (2,1,1, m-1) & i=n-1 ; \\ (1,1,1, m-1) & i=n . \\ & r\left(e_{1}^{\prime \prime} \mid A\right)=r\left(e_{5}^{\prime \prime} \mid A\right), \\ r\left(e_{1}^{\prime} \mid A\right)=r\left(e_{4}^{\prime \prime} \mid A\right), \\ r\left(e_{n-1} \mid A\right)=r\left(e_{n-2} \mid A\right) .\end{cases}
$$

All edges have different representations, which implies that $\operatorname{edim}\left(L\left(L\left(T_{n, m}\right)\right)\right) \leq 4$. On the other hand, we have to show that $\operatorname{edim}\left(L\left(L\left(T_{n, m}\right)\right)\right) \geq 4$.

Suppose to the contrary that $\operatorname{edim}\left(L\left(L\left(T_{n, m}\right)\right)\right)=3$, then we have the following possibilities. If the set $A=\left\{a_{i}, a_{j}, a_{k}\right\}$ where $i, j, k=1,2, \ldots, n$ and $i \neq j \neq k$ is an edge resolving set for graph $L\left(L\left(T_{n, m}\right)\right.$, then some edges have same representations as shown in Table 3 and in Table 4.

If the set $A=\left\{b_{s}, b_{t}, b_{u}\right\}$ where $s, t, u=2,3, \ldots, m$ and $s \neq t \neq u$ is an edge resolving set for graph $L\left(L\left(T_{n, m}\right)\right.$, then some edges have same representations as shown in Table 5.

If the set $A=\left\{a_{n}, b_{0}, b_{1}\right\}$ is an edge resolving set for graph $L\left(L\left(T_{n, m}\right)\right.$, then some edges have same representations as shown in Tables 6 and 7.

The set $A=\left\{a_{n}, b_{0}, b_{1}\right\}$ is not an edge resolving set for graph $L\left(L\left(T_{n, m}\right)\right.$ because it did not resolve the following edges:
Any combination of three vertices will not resolve the all edges of the graph $L\left(L\left(T_{n, m}\right)\right.$. Hence, there is no edge resolving set with three vertices for $L\left(L\left(T_{n, m}\right)\right.$ which implies that $e \operatorname{dim} m\left(\left(L\left(T_{n, m}\right)\right)\right) \geq 4$. So, we obtained $\operatorname{edim}(L(L$ $\left.\left.\left(T_{n, m}\right)\right)\right)=4$.

$$
\begin{aligned}
& r\left(e_{0}^{\prime} \mid A\right)=r\left(e_{2}^{\prime \prime} \mid A\right)=r\left(e_{3}^{\prime \prime} \mid A\right), \\
& r\left(e_{4}^{\prime \prime} \mid A\right)=r\left(e_{n} \mid A\right), \\
& r\left(e_{1}^{\prime \prime} \mid A\right)=r\left(e_{n-1} \mid A\right) .
\end{aligned}
$$


TABLE 3: Edge distance codes of $L\left(L\left(T_{n, m}\right)\right)$ w.r.t. $A=\left\{a_{i}, a_{j}, a_{k}\right\}$.

\begin{tabular}{lccc}
\hline$d(.,)$. & $a_{i}$ & $a_{j}$ & $a_{k}$ \\
\hline & $1 \leq i \leq\lfloor n / 2\rfloor$ & $1 \leq j \leq\lfloor n / 2\rfloor$ & $1 \leq k \leq\lfloor n / 2\rfloor$ \\
$e_{0}^{\prime}$ & $i$ & $j$ & $k$ \\
$e_{2 \prime}^{\prime \prime}$ & $i$ & $j$ & $k$ \\
$e_{3}^{\prime \prime}$ & $i$ & $j$ & $k$ \\
$e_{4}^{\prime \prime}$ & $i-1$ & $j-1$ & $k-1$ \\
$e_{n}$ & $i-1$ & $j-1$ & $k-1$ \\
\hline
\end{tabular}

TABLE 4: Edge distance codes of $L\left(L\left(T_{n, m}\right)\right)$ w.r.t. $A=\left\{a_{i}, a_{j}, a_{k}\right\}$

\begin{tabular}{lccc}
\hline$d(.,)$. & $a_{i}$ & $a_{j}$ & $a_{k}$ \\
\hline & $\lfloor n / 2\rfloor<i \leq n-1$ & $\lfloor n / 2\rfloor<j \leq n-1$ & $\lfloor n / 2\rfloor<k \leq n-1$ \\
$e_{0}^{\prime}$ & $n-i$ & $n-j$ & $n-k$ \\
$e_{2}^{\prime \prime}$ & $n-i$ & $n-j$ & $n-k$ \\
$e_{3}^{\prime \prime}$ & $n-i$ & $n-j$ & $n-k$ \\
$e_{1}^{\prime \prime}$ & $n-i-1$ & $n-j-1$ & $n-k-1$ \\
$e_{n-1}$ & $n-i-1$ & $n-j-1$ & $n-k-1$ \\
\hline
\end{tabular}

TABLE 5: Edge distance codes of $L\left(L\left(T_{n, m}\right)\right)$ w.r.t. $A=\left\{b_{s}, b_{t}, b_{u}\right\}$.

\begin{tabular}{lccc}
\hline$d(.,)$. & $b_{s}$ & $b_{t}$ & $b_{u}$ \\
\hline$e_{5}^{\prime \prime}$ & $2 \leq s \leq m-1$ & $2 \leq t \leq m-1$ & $2 \leq u \leq m-1$ \\
$e^{\prime} 1_{1}$ & $s-2$ & $t-2$ & $u-2$ \\
$e_{1}^{\prime \prime}$ & $s-2$ & $t-2$ & $u-2$ \\
$e_{2}^{\prime \prime}$ & $s-1$ & $t-1$ & $u-1$ \\
$e_{3}^{\prime \prime}$ & $s-1$ & $t-1$ & $u-1$ \\
$e_{4}^{\prime \prime}$ & $s-1$ & $t-1$ & $u-1$ \\
$e_{0}^{\prime}$ & $s-1$ & $t-1$ & $u-1$ \\
$e_{1}$ & $s-1$ & $t-1$ & $u-1$ \\
$e_{n}$ & $s$ & $t$ & $u$ \\
$e_{n-1}$ & $s$ & $t$ & $u$ \\
$e_{n-2}$ & $s$ & $t$ & $u$ \\
\hline
\end{tabular}

TABLE 6: Edge distance codes of $L\left(L\left(T_{n, m}\right)\right)$ w.r.t. $A=\left\{a_{n}, b_{0}, b_{1}\right\}$.

\begin{tabular}{lccc}
\hline$d(.,)$. & $e_{1}^{\prime \prime}$ & $e_{5}^{\prime \prime}$ & $e^{\prime} 1_{1}$ \\
\hline$A=\left\{a_{n}, b_{0}, b_{1}\right\}$ & $(1,0,1)$ & $(1,0,1)$ & $(1,1,0)$ \\
$d(.,)$. & $e_{4}^{\prime \prime}$ & $e_{n}$ & $e_{n-1}$ \\
$A=\left\{a_{n}, b_{0}, b_{1}\right\}$ & $(1,1,0)$ & $(0,1,1)$ & $(0,1,1)$ \\
\hline
\end{tabular}

TABLE 7: Comparison of the metric dimension, edge metric dimension, and mixed metric dimension.

\begin{tabular}{lcccc}
\hline & $T_{n, m}$ & $L\left(T_{n, m}\right)$ & $L\left(S\left(T_{n, m}\right)\right)$ & $L\left(L\left(T_{n, m}\right)\right)$ \\
\hline Vertex metric dimension $\operatorname{dim}(\Gamma)$ & 2 & 2 & 2 & 2 \\
Edge metric dimension $e \operatorname{dim}(\Gamma)$ & 2 & 2 & 2 & 4 \\
Mixed metric dimension $m \operatorname{dim}(\Gamma)$ & 3 & 3 & 3 & 5 \\
\hline
\end{tabular}


Theorem 15. Let $L\left(L\left(T_{n, m}\right)\right)$ be line graph of line graph of dragon graph for $n \geq 5$ and $m \geq 3$. Then, $m \operatorname{dim} L\left(\left(L\left(T_{n, m}\right)\right)\right)=5$.

Proof. Since edimL $\left(\left(L\left(T_{n, m}\right)\right)\right) \geq 3$, it is not a path graph $P_{n}$. All edges are labeled as $e_{i}=\left\{a_{i} a_{i+1}: 1 \leq i \leq n\right\}$ and $e_{j}^{\prime}=\left\{b_{j} b_{j+1}: 0 \leq j \leq m-2\right\}$.

$$
\begin{aligned}
& e_{1}^{\prime \prime}=a_{n-1} b_{0}, \\
& e_{2}^{\prime \prime}=a_{n} b_{0}, \\
& e_{3}^{\prime \prime}=a_{n} b_{1}, \\
& e_{4}^{\prime \prime}=a_{1} b_{1}, \\
& e_{5}^{\prime \prime}=b_{0} b_{2} .
\end{aligned}
$$

If $A=\left\{a_{2}, a_{\lceil n / 2\rceil+1}, b_{0}, b_{1}, b_{m-1}\right\}$ is mixed resolving set of $L\left(L\left(T_{n, m}\right)\right)$, then the representation of all edges with respect to $A$ is as follows:

$$
\begin{aligned}
& r\left(e_{j}^{\prime} \mid A\right)= \begin{cases}\left(2,\left\lfloor\frac{n}{2}\right\rfloor-1,0,0, m-2\right), & j=0 ; \\
\left(2,\left\lfloor\frac{n}{2}\right\rfloor, 1,0, m-3\right), & j=1 ; \\
\left(j+1,\left\lfloor\frac{n}{2}\right\rfloor+i-2, j-1, j-1, m-j-2\right), & 2 \leq j \leq m-2 .\end{cases} \\
& r\left(e_{1}^{\prime \prime} \mid A\right)=\left(3,\left\lfloor\frac{n}{2}\right\rfloor-2,0,1, m-2\right), \\
& r\left(e_{2}^{\prime \prime} \mid A\right)=\left(2,\left\lfloor\frac{n}{2}\right\rfloor-1,0,1, m-2\right), \\
& r\left(e_{3}^{\prime \prime} \mid A\right)=\left(2,\left\lfloor\frac{n}{2}\right\rfloor-1,1,0, m-2\right), \\
& r\left(e_{4}^{\prime \prime} \mid A\right)=\left(1,\left\lfloor\frac{n}{2}\right\rfloor, 1,0, m-2\right), \\
& r\left(e_{5}^{\prime \prime} \mid A\right)=\left(3,\left\lfloor\frac{n}{2}\right\rfloor-1,0,1, m-3\right) .
\end{aligned}
$$


For $n$ even,

$$
r\left(e_{i} \mid A\right)= \begin{cases}\left(0, \frac{n}{2}-i, 2,1, m-1\right) & i=1 ; \\ \left(i-2, \frac{n}{2}-i, i+1, i, m+i-2\right) & 2 \leq i \leq \frac{n}{2}-1 \\ \left(i-2, i-\frac{n}{2}-1, n-i-1, n-i, n+m-i-3\right) & i=\frac{n}{2} \\ \left(n-i+1, i-\frac{n}{2}-1, n-i-1, n-i, n+m-i, n+m-i-3\right) & \frac{n}{2}+2 \leq i \leq n-2 \\ \left(2, i-\frac{n}{2}-1,1,1, m-1\right) & i=n-1 ; \\ \left(1, i-\frac{n}{2}-1,1,1, m-1\right) & i=n .\end{cases}
$$

For $n$ odd,

$$
r\left(e_{i} \mid A\right)= \begin{cases}\left(0,\left\lceil\frac{n}{2}\right\rceil-i, 2,1, m-1\right) & i=1 ; \\ \left(i-2,\left\lceil\frac{n}{2}\right\rceil-i, i+1, i, m+i-2\right) & 2 \leq i \leq\left\lfloor\frac{n}{2}\right\rfloor-1 ; \\ \left(i-2,\left\lceil\frac{n}{2}\right\rceil-i, n-i-1, n-i-1, n+m-i-3\right) & i=\left\lfloor\frac{n}{2}\right\rfloor ; \\ \left(n-i+1, i-\left\lceil\frac{n}{2}\right\rceil-1, n-i-1, n-i, n+m-i-3\right) & \left.i \frac{n}{2}\right\rfloor+2 \leq i \leq n-2 ; \\ \left(2, i-\left\lceil\frac{n}{2}\right\rceil-1,1,1, m-1\right), & i=1, n+m-i-3) \\ \left(1, i-\left\lceil\frac{n}{2}\right\rceil-1,1,1, m-1\right), & i=n-1 ;\end{cases}
$$

The representation of all vertices with respect to $A$ is as follows: 


$$
\begin{aligned}
& r\left(a_{i} \mid A\right)= \begin{cases}\left(1,\left\lfloor\frac{n}{2}\right\rfloor, 2,1, m-1\right) & i=1 ; \\
\left(i-2,\left\lceil\frac{n}{2}\right\rceil-i+1, i+1, i, m+i-2\right) & 2 \leq i \leq\left\lceil\frac{n}{2}\right\rceil-1 ; \\
\left(i-2,\left\lceil\frac{n}{2}\right\rceil-i+1, n-i, i, n+m-i-2\right) & i=\left\lceil\frac{n}{2}\right\rceil ; \\
\left(i-2,\left\lceil\frac{n}{2}\right\rceil-i+1, n-i, n-i+1, n+m-i-2\right) & i=\left\lceil\frac{n}{2}\right\rceil+1 ; \\
\left(n-i+2, i-\left\lfloor\frac{n}{2}\right\rfloor-1, n-i, n-i+1, n+m-i-2\right) & \left\lceil\frac{n}{2}\right\rceil+2 \leq i \leq n-1 ; \\
\left(2,\left\lfloor\frac{n}{2}\right\rfloor-1,1,1, m-1\right), & i=n .\end{cases} \\
& r\left(b_{j} \mid A\right)= \begin{cases}\left(3,\left\lfloor\frac{n}{2}\right\rfloor-1,0,1, m-2\right), & j=0 ; \\
\left(2,\left\lfloor\frac{n}{2}\right\rfloor, 1,0, m-2\right), & j=1 ; \\
\left(j+1,\left\lfloor\frac{n}{2}\right\rfloor+j-2, j-1, j-1, m-j-1\right), & \\
2 \leq j \leq m-2 . & \end{cases}
\end{aligned}
$$

All vertices and edges have distinguished representations with respect to set $A=\left\{a_{2}, a_{\lceil n / 2\rceil+1}, b_{0}, b_{1}, b_{m-1}\right\}$, which implies that $m \operatorname{dim}\left(L\left(L\left(T_{n, m}\right)\right)\right) \leq 5$. On the other hand, we have to show that $m \operatorname{dim}\left(L\left(L\left(T_{n, m}\right)\right)\right) \geq 5$.

As we know that the edge metric dimension of line graph of line graph of dragon graph is $4, e \operatorname{dim}\left(L\left(L\left(T_{n, m}\right)\right)\right)=4$ by Theorem [6]. Let $m \operatorname{dim}\left(L\left(L\left(T_{n, m}\right)\right)\right)=4$ and set $A_{1}=\left\{a_{2}, b_{0}, b_{1}, b_{m-1}\right\}$ be mixed resolving set. The set $A_{1} \operatorname{did}$ not resolve all edges and vertices as $r\left(e_{i} \mid A_{1}\right)=r\left(a_{i} \mid A_{1}\right)$ for $2 \leq i \leq n / 2-1$ and $r\left(e_{i} \mid A_{1}\right)=r\left(a_{i+1} \mid A_{1}\right)$ for $n / 2+2 \leq$ $i \leq n-1$. That is why we have to include one more vertex $a_{\lceil n / 2\rceil+1}$ in mixed resolving set which implies that $m \operatorname{dim}\left(L\left(L\left(T_{n, m}\right)\right)\right) \geq 5$.

If the set $A_{2}=\left\{a_{2}, a_{\lceil n / 2\rceil+1}, b_{0}, b_{1}\right\}$ is mixed resolving set, then it resolves all edges and vertices of $L\left(L\left(T_{n, m}\right)\right)$. However, $r\left(e_{j}^{\prime} \mid A_{2}\right)=r\left(b_{j} \mid A_{2}\right)$ for $2 \leq j \leq m-2$. The set $A_{2}$ did not resolve all edges and vertices of $L\left(L\left(T_{n, m}\right)\right)$; this implies that $m \operatorname{dim}\left(L\left(L\left(T_{n, m}\right)\right)\right) \geq 5$.

If the set $A_{3}=\left\{a_{2}, a_{\lceil n / 2\rceil+1}, b_{1}, b_{m-1}\right\}$ is mixed resolving set of $L\left(L\left(T_{n, m}\right)\right)$, then $r\left(e_{3}^{\prime \prime} \mid A_{3}\right)=r\left(e_{0}^{\prime} \mid A_{3}\right)$; the set $A_{3} \operatorname{did}$ not resolve all edges of $L\left(L\left(T_{n, m}\right)\right)$; this implies that $m \operatorname{dim}\left(L\left(L\left(T_{n, m}\right)\right)\right) \geq 5$.

If the set $A_{4}=\left\{a_{2}, a_{\lceil n / 2\rceil+1}, b_{0}, b_{m-1}\right\}$ is mixed resolving set of $L\left(L\left(T_{n, m}\right)\right)$, then $r\left(e_{2}^{\prime \prime} \mid A_{4}\right)=r\left(e_{0}^{\prime} \mid A_{4}\right)$; the set $A_{4} \operatorname{did}$ not resolve all edges of $L\left(L\left(T_{n, m}\right)\right)$; this implies that $m \operatorname{dim}\left(L\left(L\left(T_{n, m}\right)\right)\right) \geq 5$.
If the set $A_{5}=\left\{a_{\lceil n / 2\rceil+1}, b_{0}, b_{1}, b_{m-1}\right\}$ is mixed resolving set of $L\left(L\left(T_{n, m}\right)\right)$, then $r\left(a_{n} \mid A_{5}\right)=r\left(e_{n} \mid A_{5}\right), \quad r\left(b_{0} \mid A_{5}\right)$ $=r\left(e_{2}^{\prime \prime} \mid A_{5}\right)$, and $r\left(b_{1} \mid A_{5}\right)=r\left(e_{4}^{\prime \prime} \mid A_{5}\right)$; the set $A_{5}$ did not resolve all edges and vertices of $L\left(L\left(T_{n, m}\right)\right)$; this implies that $m \operatorname{dim}\left(L\left(L\left(T_{n, m}\right)\right)\right) \geq 5$.

It is clear that $m \operatorname{dim}\left(L\left(L\left(T_{n, m}\right)\right)\right) \geq 5$ is also computed that no set of cardinality smaller than five is a mixed resolving set of $L\left(L\left(T_{n, m}\right)\right)$. So, we obtained $m \operatorname{dim}(L(L$ $\left.\left.\left(T_{n, m}\right)\right)\right)=5$.

\section{Comparison of Vertex, Edge, and Mixed Metric Dimension of Dragon Graph}

On the basis of some facts given in [17], one can easily conclude that these parameters are not comparable because there are some families graph $\Gamma$ for which $\operatorname{dim}(\Gamma)<e \operatorname{dim}(\Gamma)$, $\operatorname{dim}(\Gamma)=e \operatorname{dim}(\Gamma)$, or $\operatorname{dim}(\Gamma)>e \operatorname{dim}(\Gamma)$. Moreover, from [23], infinite families of graphs for which $\operatorname{dim}(\Gamma)>e \operatorname{dim}(\Gamma)$ is satisfied were described, which was an open problem presented in [17], where only one family satisfying this inequality was given. Any mixed metric generator is, by definition, also a metric generator and an edge metric generator. In this sense, the connection $m \operatorname{dim}(\Gamma)$ $\geq \max \{\operatorname{dim}(\Gamma), e \operatorname{dim}(\Gamma)\}[20]$ follows instantly. It is obvious that the vertex set of any graph $\Gamma$ forms a mixed metric generator for any graph $\Gamma$. In addition, any vertex of $\Gamma$, as 
well as each incident edge with it, has the same distance to the vertex itself. In this respect, a single vertex in $\Gamma$ cannot constitute a mixed metric generator.

\section{Conclusion}

In this article, we calculate the exact value of vertex, edge, and mixed dimension for dragon graph, $T_{n, m}$, line graph of dragon graph, $L\left(T_{n, m}\right)$, paraline graph of dragon graph, $L\left(S\left(T_{n, m}\right)\right)$, and line graph of line graph of dragon graph, $L\left(L\left(T_{n, m}\right)\right)$. The vertex metric dimension for $T_{n, m}, L\left(T_{n, m}\right)$, $L\left(S\left(T_{n, m}\right)\right)$, and $L\left(L\left(T_{n, m}\right)\right)$ is constant and same; $\operatorname{dim}\left(T_{n, m}\right)=\operatorname{dim}\left(L\left(T_{n, m}\right)\right)=\operatorname{dim}\left(L\left(S\left(T_{n, m}\right)\right)\right)=\operatorname{dim}$ $\left(L\left(L\left(T_{n, m}\right)\right)\right)=2$. The edge metric dimension for $T_{n, m}$, $L\left(T_{n}, m\right), L\left(S\left(T_{n, m}\right)\right)$, and $L\left(L\left(T_{n, m}\right)\right)$ is constant but $e \operatorname{dim}\left(T_{n, m}\right)=e \quad \operatorname{dim}\left(L\left(T_{n}, \quad m\right)\right)=e \operatorname{dim}\left(L\left(S\left(T_{n, m}\right)\right)\right)$ $=2<e \operatorname{dim}\left(L\left(L\left(T_{n, m}\right)\right)\right)=4$. The mixed metric dimension for $T_{n, m}, L\left(T_{n, m}\right), L\left(S\left(T_{n, m}\right)\right)$, and $L\left(L\left(T_{n, m}\right)\right)$ is constant but $m \operatorname{dim}\left(T_{n, m}\right)=m \operatorname{dim}\left(L\left(T_{n, m}\right)\right)=m \operatorname{dim}\left(L\left(S\left(T_{n, m}\right)\right)\right)$ $=3<m \operatorname{dim}\left(L\left(L\left(T_{n, m}\right)\right)\right)=5$. It is already known from [17] that there are graphs $G$ for which $\operatorname{dim}(\Gamma)<\operatorname{dim}(\Gamma)$, $\operatorname{dim}(\Gamma)=e \operatorname{dim}(\Gamma)$, or $\operatorname{dim}(\Gamma)>e \operatorname{dim}(\Gamma)$. Moreover, dragon graph, $T_{n, m}$, line graph of dragon graph, $L\left(T_{n, m}\right)$, and paraline graph of dragon graph, $L\left(S\left(T_{n, m}\right)\right)$, are families of graphs for which $\operatorname{dim}(\Gamma)=e \operatorname{dim}(\Gamma)$ but line graph of line graph of dragon graph is $L\left(L\left(T_{n, m}\right)\right) \operatorname{dim}(\Gamma)<e \operatorname{dim}(\Gamma)$.

\section{Data Availability}

All data required to prove the results are already included in the manuscript. So, no additional data are associated with this article.

\section{Conflicts of Interest}

The authors declare that there are no conflicts of interest.

\section{Authors' Contributions}

All authors contributed equally for the preparation of this article.

\section{Acknowledgments}

This research was supported by the UAEU-AUA grants of United Arab Emirates University (UAEU) via Grant no. G00003461.

\section{References}

[1] P. J. Slater, "Leaves of trees," Congressus Numerantium, vol. 14 , no. 37, pp. 549-559, 1975.

[2] F. Harary and R. A. Melter, "On the metric dimension of a graph,” Ars Combinatorics, vol. 2, no. 1, pp. 191-195, 1976.

[3] R. C. Brigham, G. Chartrand, R. D. Dutton, and P. Zhang, "Resolving domination in graphs," Mathematica Bohemica, vol. 128, no. 1, pp. 25-36, 2003.

[4] G. Chartrand, V. Saenpholphat, and P. Zhang, "The independent resolving number of a graph," Mathematica Bohemica, vol. 128, no. 4, pp. 379-393, 2003.
[5] V. Saenpholphat and P. Zhang, "Connected resolvability of graphs," Czechoslovak Mathematical Journal, vol. 53, no. 4, pp. 827-840, 2003.

[6] O. R. Oellermann and J. Peters-Fransen, "The strong metric dimension of graphs and digraphs," Discrete Applied Mathematics, vol. 155, no. 3, pp. 356-364, 2007.

[7] F. Okamoto, B. Phinezy, and P. Zhang, "The local metric dimension of a graph," Mathematica Bohemica, vol. 135, no. 3, pp. 239-255, 2010.

[8] I. G. Yero, A. Estrada-Moreno, and J. A. RodríguezVelázquez, "Computing the $\mathrm{k}$-metric dimension of graphs," Applied Mathematics and Computation, vol. 300, pp. 60-69, 2017.

[9] M. Imran and H. M. A. Siddiqui, "Computing the metric dimension of convex polytopes generated by wheel related graphs," Acta Mathematica Hungarica, vol. 149, no. 1, pp. 10-30, 2016.

[10] A. Ali, "A survey of antiregular graphs," Contributions to Mathematics, vol. 1, pp. 67-79, 2020.

[11] S. Khuller, B. Raghavachari, and A. Rosenfeld, "Landmarks in graphs," Discrete Applied Mathematics, vol. 70, no. 3, pp. 217-229, 1996.

[12] G. Chartrand, L. Eroh, M. A. Johnson, and O. R. Oellermann, "Resolvability in graphs and the metric dimension of a graph," Discrete Applied Mathematics, vol. 105, no. 1-3, pp. 99-113, 2000.

[13] R. A. Melter and I. Tomescu, "Metric bases in digital geometry," Computer Vision, Graphics, and Image Processing, vol. 25, no. 1, pp. 113-121, 1984.

[14] J. Cáceres, C. Hernando, M. Mora et al., "On the metric dimension of cartesian products of graphs," SIAM Journal on Discrete Mathematics, vol. 21, no. 2, pp. 423-441, 2007.

[15] J. Cáceres, C. Hernando, M. Mora, I. M. Pelayo, and M. L. Puertas, "On the metric dimension of infinite graphs," Discrete Applied Mathematics, vol. 160, no. 18, pp. 2618-2626, 2012.

[16] C. Hernando, M. Mora, I. M. Pelayo, C. Seara, and D. R. Wood, "Extremal graph theory for metric dimension and diameter," Electronic Journal of Combinatorics, vol. 17, no. 1,2010

[17] A. Kelenc, N. Tratnik, and I. G. Yero, "Uniquely identifying the edges of a graph: the edge metric dimension," Discrete Applied Mathematics, vol. 251, pp. 204-220, 2018.

[18] J.-B. Liu, Z. Zahid, R. Nasir, and W. Nazeer, "Edge version of metric dimension and doubly resolving sets of the necklace graph," Mathematics, vol. 6, no. 11, p. 243, 2018.

[19] B. Deng, M. Faisal Nadeem, and M. Azeem, "On the edge metric dimension of different families of Mobius networks," Mathematical Problems in Engineering, vol. 2021, Article ID 6623208, 9 pages, 2021.

[20] A. Kelenc, D. Kuziak, A. Taranenko, and G. Yero, "Mixed metric dimension of graphs," Applied Mathematics and Computation, vol. 314, pp. 429-438, 2017.

[21] I. G. Yero, "Vertices, edges, distances and metric dimension in graphs," Electronic Notes in Discrete Mathematics, vol. 55, pp. 191-194, 2016.

[22] A. Murtaza, A. Gohar, and A. Usman, "On cycle related graphs with constant metric dimension," Open Journal of Discrete Mathematics, vol. 2, no. 1, 2012.

[23] M. Knor, S. Majstorovic, A. T. M. Toshi, R. Skrekovski, and I. G. Yero, "Graphs with the edge metric dimension smaller than the metric dimension," Applied Mathematics and Computation, vol. 401, Article ID 126076, 2020. 